\title{
Flavor physics induced by light Z' from SO(10) GUT
}

\author{
Yoshihiro Shigekami ${ }^{* \dagger}$ \\ Department of Physics, Nagoya University, \\ Nagoya 464-8602, Japan \\ E-mail: sigekamideken.phys.nagoya-u.ac.jp
}

In this talk, we investigate predictions of the SO(10) Grand Unified Theory (GUT), where an extra U(1)' gauge symmetry remains up to the supersymmetry (SUSY) breaking scale. The minimal setup of $\mathrm{SO}(10)$ GUT unifies quarks and leptons into a 16-representational field in each generations. The setup, however, suffers from the realization of the realistic Yukawa couplings at the electroweak scale. In order to solve this problem, we introduce 10-representational matter fields, and then the two kinds of matter fields mix with each other at the SUSY breaking scale, where the extra U(1)' gauge symmetry breaks down radiatively. One crucial prediction is that the Standard Model quarks and leptons are given by the linear combinations of the fields with two different U(1)' charges. The mixing also depends on the flavor. Consequently, the U(1)' interaction becomes flavor violating, and the flavor physics is the smoking-gun signal of our GUT model. The flavor violating Z' couplings are related to the fermion masses and the CKM matrix, so that we can derive some explicit predictions in flavor physics. We especially discuss $K$ - $\bar{K}$ mixing, $B_{(s)}-\overline{B_{(s)}}$ mixing and the (semi)leptonic decays of $K$ and $B$ in our model. We also study the flavor violating $\mu$ and $\tau$ decays and discuss the correlations among the physical observables in this $\mathrm{SO}(10)$ GUT framework.

The 3rd International Symposium on "Quest for the Origin of Particles and the Universe" 5-7 January 2017

Nagoya University, Japan

\footnotetext{
* Speaker.

${ }^{\dagger}$ This talk is based on the work in collaboration with J. Hisano, Y. Muramatsu and Y. Omura.
} 


\section{Introduction}

In the minimal SO(10) Grand Unified Theory (GUT) model, all the Standard Model (SM) matter fields can be unified into three-family 16-representational fields[1, 2]. This means that Yukawa couplings for up-type quark, down-type quark and charged lepton are unified before the $\mathrm{SO}(10)$ gauge group are broken. This is very attractive hypothesis, but this unified Yukawa couplings cannot explain the experimental measurements of the masses of fermions and the parameters of the Cabibbo-Kobayashi-Maskawa (CKM) matrix. In order to explain these experimental measurements, many solutions have been proposed.

In this work, we focus on one of the solutions, introducing additional matter fields. In addition to the $\mathbf{1 6}$ matter fields, we introduce three-family 10 matter fields [3, 4]. The crucial point is that two kinds of SU(5) $\overline{\mathbf{5}}$-representational fields originated from $\mathbf{1 6}$ and $\mathbf{1 0}$ fields are mix with each other, and then the Yukawa couplings are modified by this mixing. To obtain the realistic Yukawa couplings, we also consider the effects of higher-dimensional operators. For this set up, an interesting point is that $Z^{\prime}$ interaction which is predicted by $\mathrm{SO}(10)$ gauge symmetry becomes flavor-dependent because these $\mathrm{SU}(5) \overline{5}$-representational fields carry different $\mathrm{U}(1)^{\prime}$ charges. Once we assume that $\mathrm{U}(1)^{\prime}$ is radiatively broken at the SUSY breaking scale, the flavor violating processes caused by $Z^{\prime}$ are verifiable in the flavor experiments.

Thus, we investigate the predictions of this model by calculating flavor violating $Z^{\prime}$ couplings quantitatively. Since our flavor violating couplings (FVCs) can induce large Flavor Changing Neutral Currents (FCNCs), we should check the consistencies with the observables related to the first and second generations, e.g. $K-\bar{K}$ mixing and lepton flavor violating (LFV) $\mu$ decays. We also investigate the predictions which is related to the third generation. Then we discuss the possibility for the observation in the future experiments.

\section{Set up and FVCs}

In our work, we introduce 10-representational chiral superfields in addition to 16-representational chiral superfields as matter. Then, we have the following superpotential for Yukawa couplings and mass terms at the renormalizable level:

$$
W=h_{i j} \mathbf{1 6}_{i} \mathbf{1 6}_{j} 1 \mathbf{0}_{H}+g_{i j} \mathbf{1 6}_{i} \mathbf{1 0}_{j} \mathbf{1 6}_{H}+\mu_{10 i j} \mathbf{1 0}_{i} 1 \mathbf{0}_{j},
$$

where $i, j=1,2,3$ is indices for the generations, $\mathbf{1 0}_{H}$ is the chiral superfield for the Higgs of minimal set up and $\mathbf{1 6}_{H}$ is an extra Higgs to break the remaining $\mathrm{U}(1)^{\prime}$ symmetry. Important point is that we have two kinds of $\overline{\mathbf{5}}$-representational fields of $\mathrm{SU}(5)$, which are originated from $\mathbf{1 6}_{i}$ and $\mathbf{1 0}_{i}$. If the scalar component of $\mathbf{1 6}_{H}$ denoted by $\Phi$ gets nonzero vacuum expectation value (VEV) $\langle\Phi\rangle \neq 0$, these fields mix with each other. As a result, we obtain the following Yukawa couplings, including the effects of higher-dimensional operators $\varepsilon c_{i j}^{d, l}$ :

$$
h_{i j}^{d}=\frac{m_{i}^{d}}{v^{d}}\left(V_{C K M}^{*}\right)_{j i}=\left(\hat{U}_{16}^{d}\right)_{i k}\left(\frac{m_{k}^{u}}{v^{u}} \delta_{k j}+\varepsilon c_{k j}^{d}\right), h_{i j}^{l}=\frac{m_{i}^{l}}{v^{d}}\left(V_{R}^{*}\right)_{j i}=\left(\hat{U}_{16}^{l}\right)_{i k}\left(\frac{m_{k}^{u}}{v^{u}} \delta_{k j}+\varepsilon c_{k j}^{l}\right)
$$

where $m_{i}^{f}(f=u, d, l)$ are the fermion masses and $v^{u}$ and $v^{d}$ are the VEV of up- and down-type Higgs doublets, respectively. $V_{R}$ is the unitary matrix and identical to the CKM matrix ( $\left.V_{\mathrm{CKM}}\right)$ in 
the $\mathrm{SU}(5)$ limit. $\left(\hat{U}_{16}^{d, l}\right)_{i j}$ are the elements of $6 \times 6$ mixing matrix for sfermion mass matrices, which determine the relation between the SM mode $\left(d_{R i}, l_{L i}\right)$ and the original fields $\left(d_{R i}^{(16),(10)}, l_{L i}^{(16),(10)}\right)$. For example, $d_{R i}=\left(\hat{U}_{16}^{d}\right)_{i j} d_{R j}^{(16)}+\left(\Delta U_{d}\right)_{i j} d_{R j}^{(10)}$, where $\left(\Delta U_{d}\right)_{i j}$ are also the elements of $6 \times 6$ mixing matrix and satisfy the unitary conditions as like $\left(\hat{U}_{16}^{d}\right)_{i k}\left(\hat{U}_{16}^{d *}\right)_{j k}+\left(\Delta U_{d}\right)_{i k}\left(\Delta U_{d}^{*}\right)_{j k}=\delta_{i j}$.

Because of mixing between two kinds of $\overline{\mathbf{5}}$-representational fields, $Z^{\prime}$ couplings with the SM mode are modified:

$$
\begin{aligned}
& \mathscr{L}_{g}=g^{\prime} \hat{Z}_{\mu}^{\prime}\left(A_{i j}^{l} \overline{l_{L}^{i}} \gamma^{\mu} l_{L}^{j}-A_{i j}^{d} \overline{d_{R}^{i}} \gamma^{\mu} d_{R}^{j}-\overline{q_{L}^{i}} \gamma^{\mu} q_{L}^{i}+\overline{u_{R}^{i}} \gamma^{\mu} u_{R}^{i}+\overline{e_{R}^{i}} \gamma^{\mu} e_{R}^{i}\right), \\
& A_{i j}^{d}=5\left(\hat{U}_{16}^{d}\right)_{i k}\left(\hat{U}_{16}^{d}\right)_{j k}^{*}-2 \delta_{i j}, A_{i j}^{l}=5\left(\hat{U}_{16}^{l}\right)_{i k}^{*}\left(\hat{U}_{16}^{l}\right)_{j k}-2 \delta_{i j},
\end{aligned}
$$

where $q_{L}^{i}, u_{R}^{i}$ and $e_{R}^{i}$ are the mass eigenstates of the left-handed quarks, right-handed up-type quarks and right-handed charged leptons. Note that the unitary conditions are used in Eq. 2.4). We can obtain the numerical values of FVCs $A_{i j}^{d, l}$ by computing $\left(\hat{U}_{16}^{d, l}\right)_{i j}$ from Eq. (2.2). In Eq. (2.3), $\hat{Z}_{\mu}^{\prime}$ is not mass eigenstate since there is the mass mixing between $\hat{Z}_{\mu}^{\prime}$ and $Z$ boson. This mixing is given by $\tan 2 \theta \simeq 4 \frac{g^{\prime}}{g_{Z}} \frac{M_{Z}^{2}}{M_{Z^{\prime}}^{2}}$.

\section{Flavor physics}

In this section, we investigate the predictions of our SO(10) GUT. In our model, there are some tree level processes induced by $Z^{\prime}$. We mainly show the results of $K-\bar{K}$ mixing, $\mu \rightarrow 3 e$ and $\mu-e$ conversion because these are tree level processes and important for our model. Some of the results for the other processes are commented in the last of this section. The details are shown in Ref. [4].

The induced operators in our model and the Wilson coefficients for these processes are

$$
\begin{aligned}
& \mathscr{H}^{K-\bar{K}}=\frac{1}{2} \widetilde{C}_{1}^{K} \widetilde{Q}_{1}^{K}=\frac{1}{2} \widetilde{C}_{1}^{K}\left(\overline{s_{R}} \gamma_{\mu} d_{R}\right)\left(\overline{s_{R}} \gamma^{\mu} d_{R}\right), \\
& \mathscr{H}^{\mu \rightarrow 3 e}=C_{L}^{3 e}\left(\overline{e_{L}} \gamma^{\mu} \mu_{L}\right)\left(\overline{e_{L}} \gamma^{\mu} e_{L}\right)+C_{R}^{3 e}\left(\overline{e_{L}} \gamma^{\mu} \mu_{L}\right)\left(\overline{e_{R}} \gamma^{\mu} e_{R}\right), \\
& \mathscr{H}^{\mu-e}=C_{q}^{\mu-e}\left(\bar{q} \gamma_{\mu} q\right)\left(\overline{e_{L}} \gamma^{\mu} \mu_{L}\right), \\
& \widetilde{C}_{1}^{K}=\left(A_{s d}^{d}\right)^{2}\left(\frac{g^{\prime 2} \cos ^{2} \theta}{M_{Z^{\prime}}^{2}}+\frac{g^{\prime 2} \sin ^{2} \theta}{M_{Z}^{2}}\right) \equiv \frac{\left(A_{s d}^{d}\right)^{2}}{\Lambda_{Z^{\prime}}^{2}} \\
& C_{L}^{3 e}=\frac{A_{e \mu}^{l}}{\Lambda_{Z^{\prime}}^{2}}\left(A_{e e}^{l}-\cos 2 \theta_{W}\right), C_{R}^{3 e}=\frac{A_{e \mu}^{l}}{\Lambda_{Z^{\prime}}^{2}}\left(1+2 \sin ^{2} \theta_{W}\right), \\
& C_{u}^{\mu-e}=\frac{A_{e \mu}^{l}}{\Lambda_{Z^{\prime}}^{2}}\left(\frac{1}{2}-\frac{4}{3} \sin ^{2} \theta_{W}\right), C_{d}^{\mu-e}=-\frac{A_{e \mu}^{l}}{\Lambda_{Z^{\prime}}^{2}}\left\{\frac{A_{d d}^{d}+1}{2}+\left(\frac{1}{2}-\frac{2}{3} \sin ^{2} \theta_{W}\right)\right\} .
\end{aligned}
$$

The physical observables are indirect CP-violation parameter $\varepsilon_{K}$ and mass difference $\Delta M_{K}$ of $K-\bar{K}$ mixing and branching ratios. These are evaluated in our model as

$$
\begin{aligned}
& \varepsilon_{K}=\frac{\kappa_{\varepsilon} e^{i \varphi_{\varepsilon}}}{\sqrt{2}\left(\Delta M_{K}\right)_{\exp }} \operatorname{Im}\left(M_{12}^{K}\right), \Delta M_{K}=2 \operatorname{Re}\left(M_{12}^{K}\right)\left(M_{12}^{K}=\left(M_{12}^{K}\right)_{\mathrm{SM}}+\frac{1}{2} \widetilde{C}_{1}^{K}(\mu)\left\langle\widetilde{Q}_{1}^{K}\right\rangle\right), \\
& \operatorname{BR}(\mu \rightarrow 3 e)=\frac{m_{\mu}^{5}}{1536 \pi^{3} \Gamma_{\mu}}\left(2\left|C_{L}^{3 e}\right|^{2}+\left|C_{R}^{3 e}\right|^{2}\right) \\
& \operatorname{BR}(\mu N \rightarrow e N)=\frac{\omega_{\text {conv }}}{\omega_{\text {capt }}}=\frac{4 m_{\mu}^{5}}{\omega_{\text {capt }}}\left|\left(2 C_{u}^{\mu-e}+C_{d}^{\mu-e}\right) V^{(p)}+\left(C_{u}^{\mu-e}+2 C_{d}^{\mu-e}\right) V^{(n)}\right|^{2}
\end{aligned}
$$



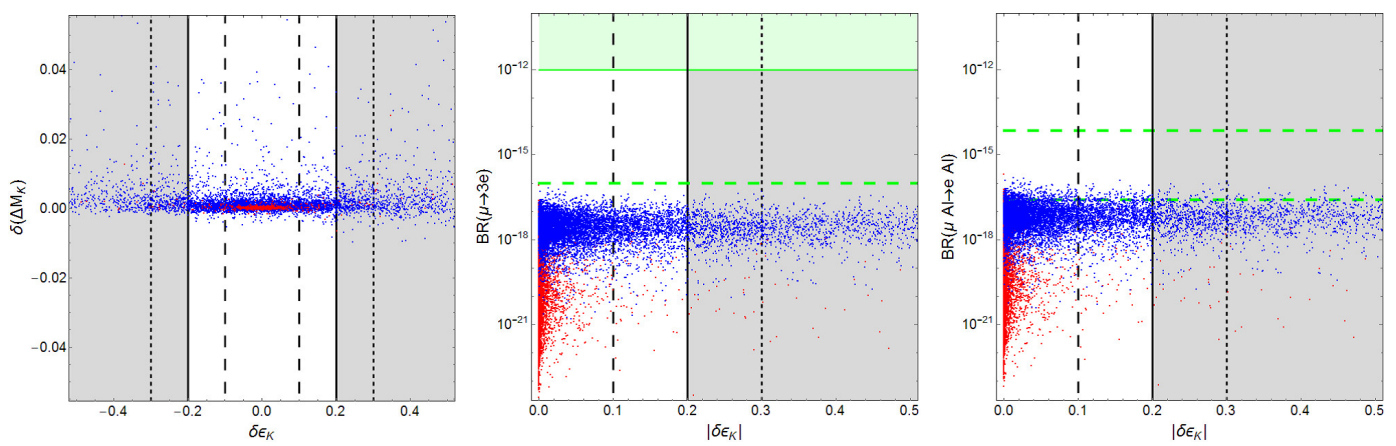

Figure 1: Our predictions for $\delta \varepsilon_{K}$ and $\delta\left(\Delta M_{K}\right)$ of $K$ meson (left panel), $\mathrm{BR}(\mu \rightarrow 3 e)$ (center panel) and $\mathrm{BR}(\mu \mathrm{Al} \rightarrow e \mathrm{Al})$ (right panel). We set $\Lambda_{Z^{\prime}}=1400 \mathrm{TeV}$. The coefficients of higher-dimensional operators satisfy $\left|\varepsilon c_{i j}^{d}\right|<10^{-2}$ (red) and $\left|\varepsilon c_{i j}^{d}\right|<10^{-3}$ (blue). In these panels, black dashed, solid and dotted line show the deviation from SM by $10 \%, 20 \%$ and $30 \%$, respectively. In the center panel, the green region is excluded by the SINDRUM experiment [7] and the green dashed line is the future prospected bound [8]. In the right panel, two green dashed lines show future sensitivity from COMET-I (upper one) and COMET-II (lower one) experiment [9,10].

The matrix element, $\left\langle\widetilde{Q}_{1}^{K}\right\rangle$, can be extracted from the SM prediction, because the only difference is the chirality. $\widetilde{C}_{1}^{K}(\mu)$ is the Wilson coefficient derived from Eq. 3.4 and renormalization group correction. $m_{\mu}$ and $\Gamma_{\mu}^{-1}$ are mass and total decay width for $\mu$, respectively. $V^{(p)}$ and $V^{(n)}$ are overlap integrals which depend on the nucleus species and $\omega_{\text {capt }}$ is the muon capture rate. These have been calculated in Ref. [5]. We use the following values for parameters: $\kappa_{\varepsilon}=0.94 \pm 0.02$, $\varphi_{\varepsilon}=0.2417 \times \pi,\left(\Delta M_{K}\right)_{\exp }=3.484(6) \times 10^{-12} \mathrm{MeV},\left|\varepsilon_{K}\right|=(2.228(11)) \times 10^{-3}, m_{\mu}=105.7 \mathrm{MeV}$ and $\Gamma_{\mu}^{-1}=2.1969811(22) \times 10^{-6} \mathrm{~s}$. 6 .

The result is shown in Fig. 1 For this figure, we set $\Lambda_{Z^{\prime}}=1.4 \times 10^{3} \mathrm{TeV}$, which corresponds to $M_{Z^{\prime}}=100 \mathrm{TeV}$ and $g^{\prime} \simeq 0.073$ [3]. The left panel of Fig. 1] shows the result for $K-\bar{K}$ mixing. In this figure, we define the deviations of the physical observables from SM predictions as $\delta \varepsilon_{K} \equiv$ $\varepsilon_{K} /\left(\varepsilon_{K}\right)_{\mathrm{SM}}-1$ and $\delta\left(\Delta M_{K}\right) \equiv \Delta M_{K} /\left(\Delta M_{K}\right)_{\mathrm{SM}}-1$. The black dashed, solid and dotted line show the deviation from SM by $10 \%, 20 \%$ and $30 \%$, respectively. From this result, we found that $\varepsilon_{K}$ largely departs from the SM prediction. Then, we have to consider the consistency with $\varepsilon_{K}$, whenever we discuss the other observables. The center and right panels of Fig. 11 show the result for $\mu \rightarrow 3 e$ and $\mu$-e conversion, respectively. $\mu \rightarrow 3 e$ has been investigated at the SIMDRUM experiment: $\operatorname{BR}(\mu \rightarrow 3 e)<1.0 \times 10^{-12}[7]$. The coming experiment will reach $\left.\mathscr{O}\left(10^{-16}\right) \llbracket 8\right]$. The predictions of our model doesn't reach the future prospected bound. On the other hand, $\mu-e$ conversion in $\mathrm{Al}$ is predicted at the range of future sensitivity from COMET-II[10] although there is no constraints in this branching ratio. Of course, these branching ratios depend on the mass of $Z^{\prime}$, so we can expect that there are some signals in future experiments for these processes when $m_{Z^{\prime}}$ is smaller than $\mathscr{O}\left(10^{2}\right) \mathrm{TeV}$. Note that in this case, $\left|\delta \varepsilon_{K}\right|$ is also enhanced.

We will comment on the other processes. Actually, our FVCs are proportional to corresponding fermion masses, so the FVCs related to 3rd generation, e.g. $A_{b s}^{d} \propto m_{s}^{d} m_{b}^{d}$, tend to be large. Then we also investigated the predictions of the other tree level processes which include 3 rd generation fermion. However, these are not so important for our model because of the largeness of the mass 
of $Z^{\prime}$. For example, the deviation for the mass difference of $B_{(s)}-\overline{B_{(s)}}$ mixing is only a few $\%$ when $\Lambda_{Z^{\prime}}=1400 \mathrm{TeV}$. When the mass of $Z^{\prime}$ is around $\mathscr{O}(10) \mathrm{TeV}$, this deviation can reach $10 \%$. The (semi)leptonic decays of $K$ and $B$ can be also induced at the tree level in our model. However, these processes are also dominated by the SM predictions even when the mass of $Z^{\prime}$ is $\mathscr{O}(10) \mathrm{TeV}$. LFV $\tau$ decays are also caused by $A_{\tau e}^{l}$ and $A_{\tau \mu}^{l}$. Although the typical values of the branching ratio of $\tau$ decays are similar to the predictions for $\mu \rightarrow 3 e$ or $\mu-e$ conversion (Fig. 1), the current bounds for these branching ratios are still large $\left(\mathscr{O}\left(10^{-(7-8)}\right)\right.$ level $)[6]$.

\section{Summary and Discussion}

In this work, we focus on $\mathrm{SO}(10)$ GUT model and its flavor physics induced by $Z^{\prime}$. Especially, we introduce 10-representational matter fields in addition to 16-representational fields and consider a certain scenario in which the SM modes are given by the linear combination of these fields. We also consider the contributions from higher-dimensional operators to the Yukawa couplings. Therefore, the realistic Yukawa couplings can be realized in our work.

The important and interesting feature of our model is that there are some FVCs of $Z^{\prime}$ caused by the field mixing and the difference of the extra $\mathrm{U}(1)^{\prime}$ charges. Then, the flavor violating $Z^{\prime}$ interactions are induced when the $\mathrm{U}(1)^{\prime}$ symmetry are spontaneously broken. Moreover, some processes are induced at the tree level and the FVCs can be $\mathscr{O}(1)$, so we can expect that the $Z^{\prime}$ contributions cause large deviation from the SM predictions. Thus we focused on the tree level processes and investigated the predictions of our model. We found that $\varepsilon_{K}$ is the most sensitive to our model. The LFV processes, such as $\mu \rightarrow 3 e$ and/or $\mu$ - $e$ conversion, are also important if the experimental bounds are improved in future.

\section{References}

[1] H. Georgi, AIP Conf. Proc. 23 (1975) 575.

[2] H. Fritzsch and P. Minkowski, Annals Phys. 93 (1975) 193.

[3] J. Hisano, Y. Muramatsu, Y. Omura and M. Yamanaka, Phys. Lett. B 744 (2015) 395 [arXiv:1503.06156 [hep-ph]].

[4] J. Hisano, Y. Muramatsu, Y. Omura and Y. Shigekami, JHEP 11 (2016) 018 [arXiv:1607.05437 [hep-ph]].

[5] R. Kitano, M. Koike and Y. Okada, Phys. Rev. D 66 (2002) 096002 [Erratum-ibid. D 76 (2007) 059902] [hep-ph/0203110].

[6] K. A. Olive et al. [Paricle Data Group], Chin. Phys. C 38 (2014) 090001.

[7] U. Bellgardt et al. [SINDRUM Collaboration], Nucl. Phys. B 299 (1988) 1.

[8] A. Blondel, A. Bravar, M. Pohl, S. Bachmann, N. Berger, M. Kiehn, A. Schoning and D. Wiedner et al., arXiv:1301.6113 [physics.ins-det].

[9] Y. Kuno [COMET Collaboration], PTEP 2013 (2013) 022 C01.

[10] COMET Collaboration,

http://comet.kek.jp/Documents_files/IPNS-Review-2014.pdf. 